\title{
Geometric phase effects in low-energy dynamics near conical intersections: A study of the multidimensional linear vibronic coupling model
}

\author{
Loïc Joubert-Doriol, ${ }^{1}$ Ilya G. Ryabinkin, ${ }^{1}$ and Artur F. Izmaylov ${ }^{1}$ \\ Department of Physical and Environmental Sciences, University of Toronto Scarborough, \\ Toronto, Ontario, M1C 1A4, Canada; and Chemical Physics Theory Group, \\ Department of Chemistry, University of Toronto, Toronto, Ontario, M5S 3H6, \\ Canada
}

(Dated: 20 September 2018)

In molecular systems containing conical intersections (CIs), a nontrivial geometric phase (GP) appears in the nuclear and electronic wave-functions in the adiabatic representation. We study GP effects in nuclear dynamics of an $N$-dimensional linear vibronic coupling (LVC) model. The main impact of GP on lowenergy nuclear dynamics is reduction of population transfer between the local minima of the LVC lower energy surface. For the LVC model, we proposed an isometric coordinate transformation that confines nonadiabatic effects within a two-dimensional subsystem interacting with an $N-2$ dimensional environment. Since environmental modes do not couple electronic states, all GP effects originate from nuclear dynamics within the subsystem. We explored when the GP affects nuclear dynamics of the isolated subsystem, and how the subsystem-environment interaction can interfere with GP effects. Comparing quantum dynamics with and without GP allowed us to devise simple rules to determine significance of the GP for nuclear dynamics in this model.

\section{INTRODUCTION}

Conical intersections (CIs) are known to play a key role in radiationless electronic transitions in molecular systems ${ }^{1}$. However, the electronic transitions are not the only features that CIs introduce in to the nuclear dynamics: Another intriguing, but much less investigated, aspect of the nuclear dynamics near CI is nontrivial geometric phase (GP) occurring in adiabatic electronic and nuclear wave-functions on encircling the CI seam ${ }^{2,3}$. The GP effects can become important even for nuclear dynamics predominantly confined to a single adiabatic electronic surface ${ }^{4}$. For example, charge and energy transfer processes considered in the adiabatic representation may not go far beyond a single electronic surface description, and thus, can experience significant GP effects in the presence of $\mathrm{CI}^{5-7}$ (see Fig. 1). As we have shown in our previous paper ${ }^{4}$, the GP has a significant impact on the low-energy nuclear dynamics of systems with CI: tunnelling of a localized nuclear wave-packet from one well to another is significantly reduced or even blocked completely in the presence of GP. This is a result of destructive interference between parts of the initial wave packet traveled on different sides from the CI (Fig. 1). The same interference effect causes a nodal line to appear in the tunnelled wave packet ${ }^{8,9}$.

Most of the previous studies of GP were done on small models ${ }^{4,9-11}$ or molecular systems with a few atoms ${ }^{12-14}$, therefore, it is still unclear how GP effects can modify dynamics of a large multidimensional system with CI. Usually quantum effects diminish with increase of the system size, and GP effects as purely quantum are expected to follow this trend. Study of Kelly and Kapral ${ }^{15}$ supported this view by illustrating that the associated with GP nodal line in a subsystem adiabatic nuclear density disappears after including interaction with an envi-

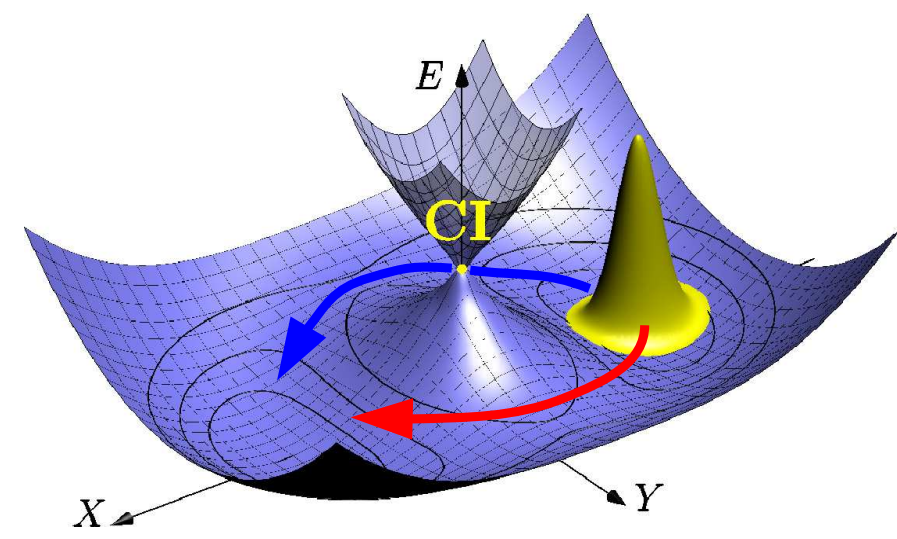

FIG. 1. Destructive interference due to geometric phase in low energy dynamics. The minimum on the $X<0(X>0)$ side can correspond to the donor (acceptor) state for charge or energy transfer processes. ${ }^{5,6}$

ronment. On the other hand, studies on two-level spin subsystems coupled to environment ${ }^{16,17}$ suggest that GP effects survive, and thus, the GP can be used to encode information in quantum computing. Considering these seemingly controversial results from two communities, we would like to assess GP effects in large vibronic multidimensional systems with CIs. The difference of our approach is to consider not only the nodal line in the nuclear density as a sign of GP but also to compare population dynamics in simulations with and without GP effects. The latter comparison is mostly motivated by the idea that the nodal line is one of the consequences of GP effects but its absence, in general, cannot be considered as a sign of GP insignificance.

To study GP effects in multidimensional systems, we consider the $N$-dimensional linear vibronic coupling 
(LVC) Hamiltonian model ${ }^{18}$

$$
\begin{gathered}
H_{\mathrm{LVC}}=\sum_{j}^{N} \frac{1}{2}\left(p_{j}^{2}+\omega_{j}^{2} q_{j}^{2}\right) \mathbf{1}_{2}+\left(\begin{array}{cc}
\kappa_{j} q_{j} & c_{j} q_{j} \\
c_{j} q_{j} & \tilde{\kappa}_{j} q_{j}
\end{array}\right) \\
+\left(\begin{array}{cc}
-\delta / 2 & 0 \\
0 & \delta / 2
\end{array}\right)
\end{gathered}
$$

where we use mass-weighted coordinates $q_{j}$ and their conjugated momenta $p_{j}, \omega_{j}$ are the frequencies, $\kappa_{j}, \tilde{\kappa}_{j}$, and $c_{j}$ are linear coupling constants. $\delta$ is the energy difference between the minima of the two diabatic electronic potentials. All quantities in this equation are given in atomic units, which are used throughout this work. In spite of its simplicity, $H_{\mathrm{LVC}}$ has been successfully applied to model vibronic spectra of various molecular systems (e.g., Jahn-Teller distorted molecules) ${ }^{19-21}$ and used as an ansatz for approximate diabatization methods ${ }^{7,22,23}$.

Another advantage of the LVC model found in the course of this work is existence of an isometric transformation that maps the $N$-dimensional $H_{\text {LVC }}$ into a Hamiltonian where all non-adiabatic effects are confined within a two-dimensional branching subspace spanned by two collective nuclear coordinates (CNC). The other $N-2$ $\mathrm{CNC}$ can be seen as environmental degrees of freedom (DOF) that interacts with the CNC of the branching subspace identically for both electronic states. Our transformation is similar to the ones found earlier by Cederbaum and coworkers ${ }^{24-26}$ with the main difference that all previous transformations had introduced three-dimensional electronically coupled subsystems while ours has only a two-dimensional subsystem. Thus, we employ the methods developed to analyze GP effects in the 2D-LVC prob$\mathrm{lem}^{4}$ and augment them by time-convolutionless master equation approach ${ }^{27}$ to account for the subsystemenvironment interaction. Owing to the isometric transformation that confines non-adiabatic effects within the branching subspace, multimode consideration of GP effects in $H_{\text {LVC }}$ is split in two steps: 1) GP effects within the branching subspace, and 2) influence of subsystemenvironment interaction on GP effects. This split allows us to formulate simple rules on when GP effects are expected to be important in the $N$-dimensional LVC model.

The rest of the paper is organized as follows. In Section II we illustrate the origin of the GP on a twodimensional LVC model. Section III describes the LVC isometric transformation and techniques used to simulate the nuclear non-adiabatic dynamics. Section IV provides qualitative analysis of the nuclear non-adiabatic dynamics for several variations of system parameters. Section V discusses results of the nuclear dynamics with and without the GP for isolated subsystem and subsystem interacting with its environment. Section VI concludes by summarizing our main findings.

\section{MOTIVATING EXAMPLE: TWO-DIMENSIONAL LVC MODEL}

To illustrate importance of GP effects we consider the simplest two-dimensional LVC model where a nontrivial GP appears. The model Hamiltonian is

$$
H_{2 \mathrm{D}}=T_{2 \mathrm{D}} \mathbf{1}_{2}+\left(\begin{array}{ll}
V_{11} & V_{12} \\
V_{12} & V_{22}
\end{array}\right)
$$

where $T_{2 \mathrm{D}}=-1 / 2\left(\partial^{2} / \partial x^{2}+\partial^{2} / \partial y^{2}\right)$ is the nuclear kinetic energy operator, $x$ and $y$ are the mass-weighted coordinates, $V_{11}$ and $V_{22}$ are the diabatic potentials represented by identical two-dimensional parabolas shifted in space and coupled by the $V_{12}$ potential

$$
\begin{aligned}
& V_{11}=\frac{\omega^{2}}{2}\left[\left(x+x_{0}\right)^{2}+y^{2}\right], \quad V_{12}=c y, \\
& V_{22}=\frac{\omega^{2}}{2}\left[\left(x-x_{0}\right)^{2}+y^{2}\right] .
\end{aligned}
$$

Here, $\omega$ is the frequency for both coordinates, $\pm x_{0}$ are the minima of $V_{11}$ and $V_{22}$ potentials, and $c$ is a coupling constant. $H_{2 \mathrm{D}}$ is an electron-nuclear Hamiltonian written in the so-called diabatic representation with the nuclear kinetic energy operator $T_{2 \mathrm{D}} \mathbf{1}_{2}$ diagonal in the electronic subspace. Electronic DOF in $H_{2 \mathrm{D}}$ are abstract vectors $|1\rangle$ and $|2\rangle$ in a two-dimensional linear space. Both nonadiabatic transitions and GP effects are accounted in $H_{2}$ D implicitly via the off-diagonal elements of the potential matrix $V_{12}$. To obtain the corresponding adiabatic representation of the Hamiltonian one needs to diagonalize the two-state potential matrix in Eq. (2) by unitary transformation

$$
U(\theta)=\left(\begin{array}{rr}
\cos \frac{\theta}{2} & -\sin \frac{\theta}{2} \\
\sin \frac{\theta}{2} & \cos \frac{\theta}{2}
\end{array}\right),
$$

where $\theta$ is a mixing angle between the diabatic states $|1\rangle$ and $|2\rangle$ defined as

$$
\theta=\arctan \frac{2 V_{12}}{V_{11}-V_{22}} .
$$

The diabatic-to-adiabatic transformation $U(\theta)$ defines the adiabatic electronic states

$$
\begin{aligned}
\left|\phi_{1}^{\text {adi }}\right\rangle & =\cos \frac{\theta}{2}|1\rangle+\sin \frac{\theta}{2}|2\rangle \\
\left|\phi_{2}^{\text {adi }}\right\rangle & =-\sin \frac{\theta}{2}|1\rangle+\cos \frac{\theta}{2}|2\rangle
\end{aligned}
$$

and brings the Hamiltonian (2) to the form

$$
H_{2 \mathrm{D}}^{\mathrm{adi}}=\left(\begin{array}{cc}
T_{2 \mathrm{D}}+\tau_{11} & \tau_{12} \\
\tau_{21} & T_{2 \mathrm{D}}+\tau_{22}
\end{array}\right)+\left(\begin{array}{cc}
W_{1} & 0 \\
0 & W_{2}
\end{array}\right)
$$

where

$$
W_{1,2}=\frac{1}{2}\left(V_{11}+V_{22}\right) \mp \frac{1}{2} \sqrt{\left(V_{11}-V_{22}\right)^{2}+4 V_{12}^{2}},
$$


are the adiabatic potentials with the minus (plus) sign for $W_{1}\left(W_{2}\right), \tau_{i j}=-\left\langle\phi_{i}^{\text {adi }} \mid \nabla \phi_{j}^{\text {adi }}\right\rangle \cdot \nabla-\frac{1}{2}\left\langle\phi_{i}^{\text {adi }} \mid \nabla^{2} \phi_{j}^{\text {adi }}\right\rangle$ are non-adiabatic couplings with $\nabla=(\partial / \partial x, \partial / \partial y)$.

In the adiabatic representation, if nuclei undergo infinitely slow (adiabatic) evolution around the CI point, $\theta$ changes from 0 to $2 \pi$. Since $U(2 \pi)=-\mathbf{1}_{2}$, both adiabatic electronic states $\left\{\left|\phi_{i}^{\text {adi }}\right\rangle\right\}_{i=1}^{2}$ change their signs after encircling the CI. This sign change is the result of acquisition of the nontrivial GP during the adiabatic evolution around a degeneracy point ${ }^{2,3}$. This also means that both adiabatic electronic states $\left\{\left|\phi_{i}^{\text {adi }}\right\rangle\right\}_{i=1}^{2}$ are double-valued functions of nuclear coordinates, and that the CI point is a branching point for them.

Hamiltonians (2) and (9) should produce exactly the same nuclear dynamics because they are connected by the unitary transformation $U(\theta)$. However, the nuclear wavefunctions associated with $H_{2 \mathrm{D}}$ and $H_{2 \mathrm{D}}^{\text {adi }}$ are subjected to different boundary conditions: In the diabatic representation both nuclear and electronic wavefunctions are single-valued. In the adiabatic representation the electronic wave functions are double-valued, and thus, to have a single-valued total wavefunction, one has to impose the double-valued boundary condition on the nuclear wave functions $\left\{\left|\chi_{j}^{\text {adi }}\right\rangle\right\}_{j=1}^{2}$. Double-valued boundary conditions can be cumbersome to implement practically. To address this issue Mead and Truhlar ${ }^{3}$ proposed to factorize the double-valued adiabatic nuclear wavefunctions as $\left|\chi_{j}^{\text {adi }}\right\rangle=e^{i \gamma}\left|\tilde{\chi}_{j}^{\text {adi }}\right\rangle$, where $\gamma$ is a function that changes from 0 to $\pi$ along a path encircling a CI seam and $\left|\tilde{\chi}_{j}^{\text {adi }}\right\rangle$ are single-valued functions. The factor $e^{i \gamma}$ is the geometric phase, and for our 2D model it can be expressed as $\gamma=\theta / 2$, since $\theta$ changes by $2 \pi$ upon encircling the CI point. Neglecting the GP or doublevaluedness of the nuclear wavefunctions in the adiabatic representation can result in drastically different nuclear dynamics as shown in Fig. 2. Thus, it is not sufficient to account only for the non-adiabatic couplings $\tau_{i j}$ [Eq. (9)] to obtain the correct dynamics in the adiabatic representation but the double-valuedness or GP of the nuclear wavefunction must also be included. The GP $e^{i \gamma}$ makes parts of a nuclear wave packet traveling on different sides from the CI point to acquire the opposite phases $e^{ \pm i \pi / 2}$ (red and blue paths in Fig. 1). This results in the destructive interference between these parts and gives rise to a nodal line in the adiabatic nuclear wave packet (Fig. 3a). The GP origin of the nodal line can be verified by simulating nuclear dynamics without GP where the nodal line does not appear (Fig. 3b).

\section{METHOD}

\section{A. Model Hamiltonian}

To generalize the consideration of the GP in the 2D LVC model to the $N$-dimensional case we propose a series of transformations (detailed in the Appendix A) that bring the $N$-dimensional LVC Hamiltonian to the follow-

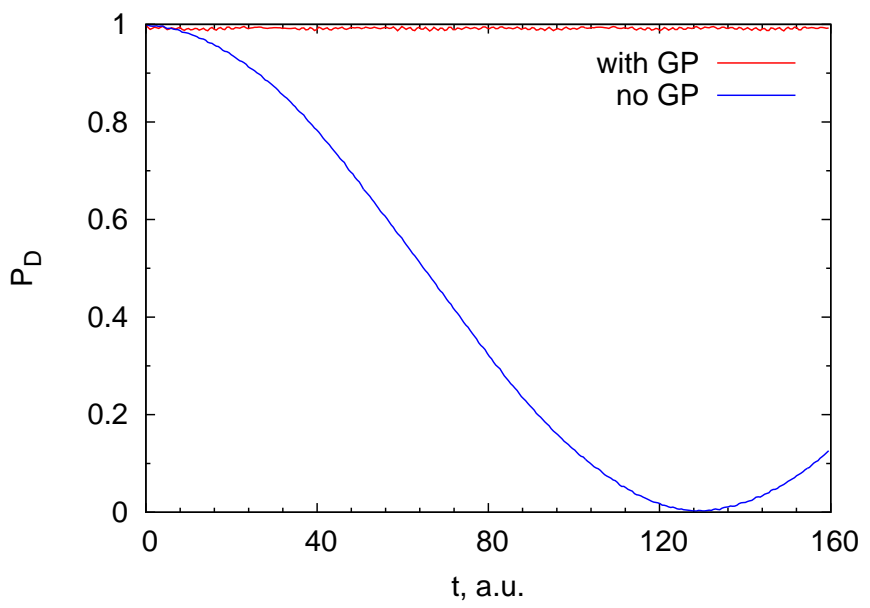

FIG. 2. Population transfer between adiabatic wells: $P_{D}$ is a fraction of nuclear density corresponding to negative $x$ values. Parameters of Eqs. (3) and (4) used here are $\omega=2, c=3$, $x_{0}=1.5$ in a.u. The initial wavefunction is $|\chi\rangle\left|\phi_{1}^{\text {adi }}\right\rangle$, where $|\chi\rangle$ is the ground vibrational state of the Hamiltonian $T_{2 \mathrm{D}}+$ $V_{11}$. a)

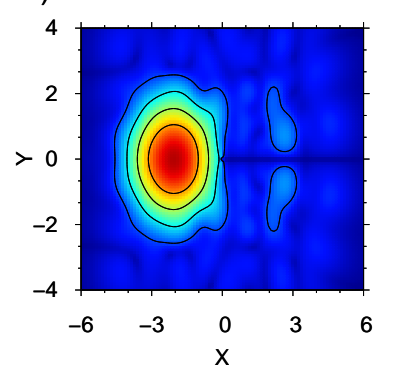

b)

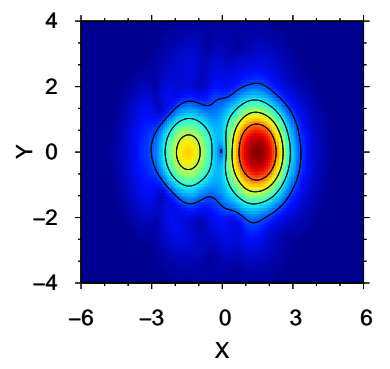

FIG. 3. Snapshots of $\sqrt{\left|\chi_{1}^{\text {adi }}\right|}$ at $t=100.0$ a.u. for the same parameters as in Fig. 2: a) with GP, and b) without GP. The square root is used to make a nodal line legible.

ing equivalent form

$$
H=H_{\mathrm{S}}+H_{\mathrm{SB}}+H_{\mathrm{B}},
$$

with

$$
\begin{aligned}
& H_{\mathrm{S}}=T_{\mathrm{S}} \mathbf{1}_{2}+\left(\begin{array}{cc}
V_{A} & V_{c} \\
V_{c} & V_{D}
\end{array}\right), \\
& H_{\mathrm{B}}=\frac{1}{2} \sum_{j=1}^{N-2}\left(P_{j}^{2}+\Omega_{j}^{2} Q_{j}^{2}\right) \mathbf{1}_{2}, \\
& H_{\mathrm{SB}}=\sum_{j=1}^{N-2}\left(\lambda_{j X} X+\lambda_{j Y} Y\right) Q_{j} \mathbf{1}_{2} .
\end{aligned}
$$




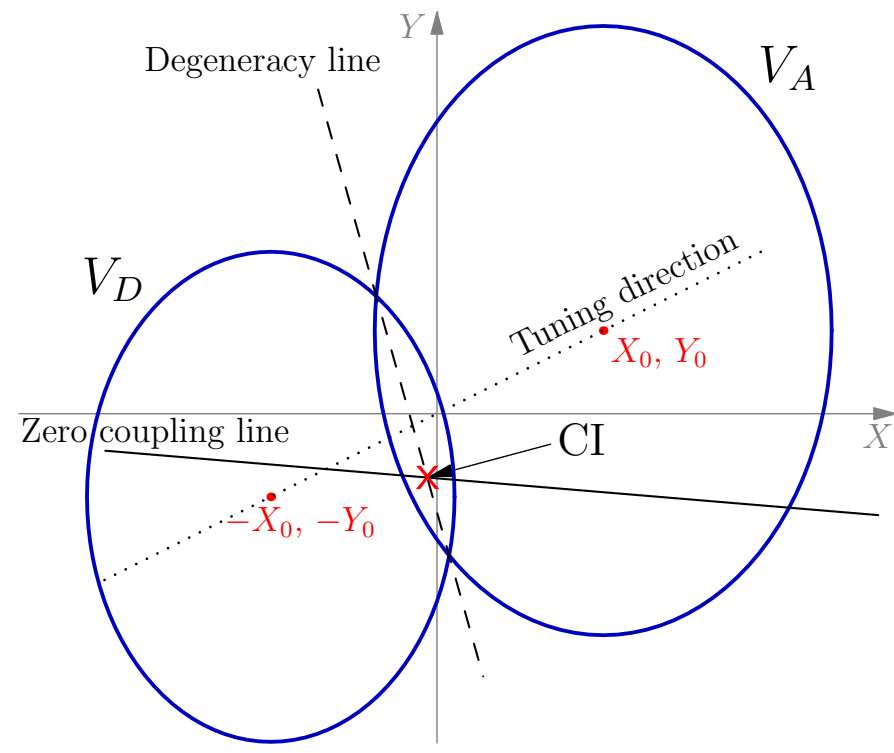

FIG. 4. The geometry of the subsystem Hamiltonian (12): the important elements that accompanied the conical intersection (terminology is explained in the text) in a constant-energy ( $E=7$ a.u.) plane. The Hamiltonian parameters are: $\Omega_{X}=$ $2, \Omega_{Y}=3 / 2, \mathbf{R}_{0}=(3 / 2,3 / 4), \Delta=3, \mathbf{C}=(1 / 4,3), \Delta_{12}=$ $-7 / 4$.

In this form all non-adiabatic effects are confined in the two-dimensional subsystem Hamiltonian $H_{\mathrm{S}}$ with

$$
\begin{aligned}
T_{\mathrm{S}} & =\frac{1}{2}\left(P_{X}^{2}+P_{Y}^{2}\right), \\
V_{D} & =\frac{1}{2}\left[\Omega_{X}^{2}\left(X+X_{0}\right)^{2}+\Omega_{Y}^{2}\left(Y+Y_{0}\right)^{2}+\Delta\right] \\
V_{A} & =\frac{1}{2}\left[\Omega_{X}^{2}\left(X-X_{0}\right)^{2}+\Omega_{Y}^{2}\left(Y-Y_{0}\right)^{2}-\Delta\right] \\
V_{c} & =C_{X} X+C_{Y} Y+\Delta_{12}
\end{aligned}
$$

where $X$ and $Y$ are the subsystem's CNC, $P_{X}$ and $P_{Y}$ are corresponding momenta. The parameters involved in the potentials $V_{D}, V_{A}$, and $V_{c}$ are functions of the LVC Hamiltonian parameters and have the following geometrical meaning

- Vector $\mathbf{R}_{0}=\left(X_{0}, Y_{0}\right)$ determines a line that connects the $V_{D}$ and $V_{A}$ minima and is referred as a tuning direction (Fig. 4).

- Vector $\mathbf{G}=\left(G_{X}, G_{Y}\right)=\left(\Omega_{X}^{2} X_{0}, \Omega_{Y}^{2} Y_{0}\right)$ is a normal vector to the degeneracy line where $V_{D}=V_{A}$ (Fig. 4).

- Vector $\mathbf{C}=\left(C_{X}, C_{Y}\right)$ determines a coupling direction and is a normal vector to a zero coupling line where $V_{c}=0$ (Fig. 4).

- Parameter $\Delta$ is the energy difference between the $V_{D}$ and $V_{A}$ minima.

- Parameter $\Delta_{12}$ determines the displacement of the zero coupling line along the $\mathbf{C}$ vector.
The subsystem coordinates $X$ and $Y$ interact with $N-2$ harmonic modes $Q_{j}$ of $H_{\mathrm{B}}$ through the diagonal in the electronic subspace subsystem-bath Hamiltonian $H_{\mathrm{SB}}$ with coupling constants $\lambda_{j X}$ and $\lambda_{j Y}$.

For further discussion, we define a special symmetric subsystem setup that corresponds to the Hamiltonian (2): $Y_{0}=\Delta=\Delta_{12}=C_{X}=0$. For this setup both the tuning direction and the zero coupling line coincide with the $X$ direction, whereas the degeneracy line is orthogonal to the tuning direction and the zero coupling line.

\section{B. Dynamics of isolated subsystem}

To assess importance of the GP for the $H_{\mathrm{S}}$ Hamiltonian we model nuclear dynamics in both diabatic and adiabatic representations. The diabatic Hamiltonian $H_{S}^{\text {dia }}=H_{S}$ is already given by Eqs. (11)-(14) while the adiabatic Hamiltonian $H_{S}^{\text {adi }}$ is obtained through diagonalization of the two-state potential matrix in Eq. (12). The propagation of the subsystem density is done using the unitary evolution $\rho_{S}(t)=e^{-i H_{S} t} \rho_{S}(0) e^{i H_{S} t}$ after projecting $\rho_{S}(t)$ and $H_{S}$ on a finite basis set. The choice of the basis set depends on the representation of the subsystem Hamiltonian $H_{S}^{\text {adi }}$ or $H_{S}^{\text {dia }}$ and is explained in the supplemental material. ${ }^{28}$ All employed basis functions are single-valued, and thus, effects of the GP are included in the diabatic representation and are neglected in the adiabatic representation. Note that the non-adiabatic transitions between two electronic states are included in both representations.

To monitor the nuclear dynamics we calculate the time evolution of a projected subsystem population $P_{D}(t)=$ $\operatorname{Tr}\left\{\rho_{S}(t) \hat{P}_{D}\right\}$, where $\hat{P}_{D}$ is the projector on the donor well which is defined as $\hat{P}_{D}=1$ for all $X, Y$ in the left side from the degeneracy line of Fig. 4 , and $\hat{P}_{D}=0$ otherwise. The initial state of $\rho_{S}(t)$ is $|\chi\rangle\left|\varphi_{1}^{\text {adi }}\right\rangle\left\langle\varphi_{1}^{\text {adi }}\right|\langle\chi|$, where $\left|\varphi_{1}^{\text {adi }}\right\rangle$ is the ground adiabatic state of $H_{S}$, and $|\chi\rangle$ is the ground vibrational state of the Hamiltonian $T_{S}+V_{D}$.

\section{Dynamics with environment}

To account for the interaction $H_{\mathrm{SB}}$ between the subsystem and a large number of bath DOF we follow a timeconvolutionless master equation (TCLME) approach to reduced subsystem dynamics that accounts for the interaction up to a second order in $H_{\mathrm{SB}}{ }^{27}$. Starting from the Liouville-von Neumann equation for the total density of the system

$$
\frac{\partial}{\partial t} \rho(t)=-i[H, \rho(t)]
$$

and using standard thermal projectors ${ }^{27}$ one can integrate out the bath DOF assuming that in the zeroth order the bath density is Boltzmann $\rho_{B}^{T}=$ 
$\exp \left(-\frac{H_{\mathrm{B}}}{k T}\right) / \operatorname{Tr}\left\{\exp \left(-\frac{H_{\mathrm{B}}}{k T}\right)\right\}$, and there is no initial correlation between the subsystem and environment $\rho(0)=$ $\rho_{S}(0) \rho_{B}^{T}$. For our subsystem-bath interaction $H_{\mathrm{SB}}$, TCLME is

$$
\begin{aligned}
& \frac{\partial}{\partial t} \rho_{S}(t)=-i\left[H_{S}, \rho_{S}(t)\right] \\
& -\sum_{j}\left[\left(\lambda_{j X} X+\lambda_{j Y} Y\right),\left(\lambda_{j X} X_{j}(t)+\lambda_{j Y} Y_{j}(t)\right) \rho_{S}(t)\right] \\
& -\sum_{j}\left[\rho_{S}(t)\left(\lambda_{j X} X_{j}^{\dagger}(t)+\lambda_{j Y} Y_{j}^{\dagger}(t)\right),\left(\lambda_{j X} X+\lambda_{j Y} Y\right)\right],
\end{aligned}
$$

where $X_{j}(t)$ and $Y_{j}(t)$ are the $X$ and $Y$ operators dressed by time-dependent functions

$$
\begin{aligned}
& X_{j}(t)=\int_{0}^{t} d t^{\prime} e^{-i H_{\mathrm{S}} t^{\prime}} X e^{i H_{\mathrm{S}} t^{\prime}}\left\langle Q_{j}(0) Q_{j}\left(t^{\prime}\right)\right\rangle_{T}, \\
& Y_{j}(t)=\int_{0}^{t} d t^{\prime} e^{-i H_{\mathrm{S}} t^{\prime}} Y e^{i H_{\mathrm{S}} t^{\prime}}\left\langle Q_{j}(0) Q_{j}\left(t^{\prime}\right)\right\rangle_{T} .
\end{aligned}
$$

Here, $\langle\ldots\rangle_{T}$ is a thermal average over the bath coordinates with the bath Boltzmann density $\rho_{B}^{T}$. The bath correlators $\left\langle Q_{j}(0) Q_{j}\left(t^{\prime}\right)\right\rangle_{T}$ can be evaluated analytically ${ }^{27}$

$$
\left\langle Q_{j}(0) Q_{j}\left(t^{\prime}\right)\right\rangle_{T}=\frac{1}{2 \Omega_{j}}\left(e^{-i \Omega_{j} t^{\prime}}+\frac{2 \cos \left(\Omega_{j} t^{\prime}\right)}{e^{\frac{\Omega_{j}}{k T}}-1}\right) .
$$

The propagation of the $\rho_{S}(t)$ is done numerically after projecting the density matrix and $H_{\mathrm{S}}$ on a finite basis set in the diabatic or adiabatic representations. The diabatic-to-adiabatic transformation does not modify the $H_{\mathrm{B}}$ and $H_{\mathrm{SB}}$ Hamiltonians, and thus, all parts of Eq. (20) describing the subsystem-bath interaction are invariant of the electronic representation.

The parameters of the harmonic bath are generated by an Ohmic spectral density ${ }^{29}$

$$
J(\Omega)=\pi \sum_{j=1}^{N-2} \frac{\lambda_{j}^{2}}{2 \Omega_{j}} \delta\left(\Omega-\Omega_{j}\right)
$$

where $\lambda_{j}=\Omega_{j} \sqrt{\xi \Omega_{0}}, \Omega_{j}=-\Omega_{c} \ln \left(1-j \Omega_{0} / \Omega_{c}\right)$, and $\Omega_{0}=\Omega_{c}\left(1-e^{\Omega_{\max } / \Omega_{c}}\right) /(N-2)$. The bath is characterized by a cut-off frequency $\Omega_{c}$ that determines the peak and width of $J(\Omega)$, and a Kondo parameter $\xi$ that characterizes the overall subsystem-bath coupling strength. In our simulations we couple only one subsystem coordinate to the bath modes therefore one Kondo parameter generating either $\lambda_{j X}=\lambda_{j}$ or $\lambda_{j Y}=\lambda_{j}$ is sufficient. As in Ref. 15, we use $N-2=100$ bath DOF, and the highest frequency of the discrete bath $\Omega_{\max }=3 \Omega_{c}$. Temperature of the bath is fixed to $0 \mathrm{~K}$ in all simulations to avoid technical complications with the basis size set in the subsystem dynamics.

\section{QUALITATIVE ANALYSIS}

Before considering the numerical simulations we would like to present some qualitative analysis of the population

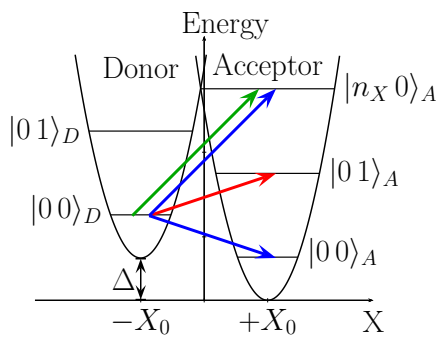

FIG. 5. Main channels for population transfer in the diabatic representation for a non-symmetric isolated subsystem: i) the red arrow for $\Delta \neq 0$, ii) the blue arrows for $\Delta_{12} \neq 0$, iii) the green arrow for $C_{X} \neq 0$.

transfer dynamics and the $Y=0$ nodal line emergence for the isolated and coupled to the environment subsystems. Our analysis is based on the time-dependent perturbation theory (TDPT) in the diabatic representation that takes the population on the donor diabatic state $\left(V_{D}\right)$ as a measure of the population transfer. Although this measure is formally different from $P_{D}(t)$ defined earlier it is qualitatively the same for low-energy dynamics. For simplicity, we consider pure initial states $|00\rangle_{D}$ for the isolated subsystem and $|00 \mathbf{n}\rangle_{D}$ for the full system. Here, we use the state notations $\left|n_{X} n_{Y}\right\rangle_{e}$ and $\left|n_{X} n_{Y} \mathbf{n}\right\rangle_{e}$, where $e=D, A$ is an electronic state, $n_{X}$ and $n_{Y}$ are the numbers of vibrational quanta on the $X$ and $Y$ modes, and $\mathbf{n}$ is a vector of vibrational quanta on the bath modes $Q_{j}$. The subsystem is assumed to have isotropic parabolas $\Omega_{X}=\Omega_{Y}=\Omega$ with $\mathbf{R}_{0}=(R / 2,0)$ and $\mathbf{C} \cdot \mathbf{R}_{0}=0$ [Eqs. (16) and (17)].

\section{A. Isolated subsystem}

The subsystem Hamiltonian (12) is partitioned as $H_{\mathrm{S}}=H_{0}+V$ with

$$
\begin{aligned}
H_{0} & =T_{\mathrm{S}} \mathbf{1}_{2}+\left(\begin{array}{cc}
V_{A} & 0 \\
0 & V_{D}
\end{array}\right), \\
V & =\left(\begin{array}{cc}
0 & V_{c} \\
V_{c} & 0
\end{array}\right) .
\end{aligned}
$$

In the first order of TDPT, the population transfer for the symmetric setup considered in Sec. III A is

$$
P_{D \rightarrow A}^{(1, a)}(t)=\left|{ }_{A}\left\langle 01\left|C_{Y} Y \sigma_{x}\right| 00\right\rangle_{D}\right|^{2} \frac{\sin ^{2}(\Omega t / 2)}{(\Omega / 2)^{2}} .
$$

This expression explains both the origin of the nodal line due to the wave-function parity change along the $Y$ direction and slow (almost frozen) population transfer in Fig. 2 due to the damping prefactor $\left|{ }_{A}\left\langle 01\left|C_{Y} Y \sigma_{x}\right| 00\right\rangle_{D}\right|^{2} / \Omega^{2}$. The population transfer changes if we depart from the symmetric setup. Here, we will consider the following symmetry breaking scenarios: i) $\Delta \neq 0$, ii) $\Delta_{12} \neq 0$, and iii) $C_{X} \neq 0$. 
(i) Energy bias $\Delta \neq 0$ (Fig. 5) results in modification of the population transfer as

$$
\begin{aligned}
P_{D \rightarrow A}^{(1, a)}(t) & =\left|{ }_{A}\left\langle 01\left|C_{Y} Y \sigma_{x}\right| 00\right\rangle_{D}\right|^{2} \\
& \times \frac{\sin ^{2}[(\Omega-\Delta) t / 2]}{[(\Omega-\Delta) / 2]^{2}} .
\end{aligned}
$$

Qualitative difference between the population dynamics in Eqs. (28) and (27) occurs for the resonance condition $\Delta=\Omega$, where the population transfer in Eq. (28) can be further simplified as

$$
\lim _{\Delta \rightarrow \Omega} P_{D \rightarrow A}^{(1, a)}(t)=\left.\left.\right|_{A}\left\langle 01\left|C_{Y} Y \sigma_{x}\right| 00\right\rangle_{D}\right|^{2} t^{2} .
$$

The resonance strongly facilitates the population flow and corresponds to the isoenergetic position of $H_{0}$ vibronic levels that are coupled by $V_{c}$.

(ii) Constant coupling $\Delta_{12} \neq 0$ (Fig. 5) leads to opening another population transfer channel that in the first order contributes as

$$
\begin{aligned}
P_{D \rightarrow A}^{(1, b)}(t) & =\Delta_{12}^{2} \sum_{n_{X}=0}^{\infty}\left|{ }_{A}\left\langle n_{X} 0\left|\sigma_{x}\right| 00\right\rangle_{D}\right|^{2} \\
& \times \frac{\sin ^{2}\left(n_{X} \Omega t / 2\right)}{\left(n_{X} \Omega / 2\right)^{2}} .
\end{aligned}
$$

Here, the infinite summation over all vibronic levels with $n_{Y}=0$ is due to a finite shift along the $X$ coordinate between the $V_{D}$ and $V_{A}$ minima. The resonance condition is satisfied for the $n_{X}=0$ term

$$
\begin{aligned}
& \left.\Delta_{12}^{2}\left|{ }_{A}\left\langle n_{X} 0\left|\sigma_{x}\right| 00\right\rangle_{D}\right|^{2} \frac{\sin ^{2}\left(n_{X} \Omega t / 2\right)}{\left(n_{X} \Omega / 2\right)^{2}}\right|_{n_{X}=0}= \\
& \left.\left.\Delta_{12}^{2}\right|_{A}\left\langle 01\left|\sigma_{x}\right| 00\right\rangle_{D}\right|^{2} t^{2}
\end{aligned}
$$

therefore, the population transfer is dominated by this term. Opening of the $\Delta_{12} \neq 0$ channel provides the population flow that has a maximum at $Y=0$ line, and thus, its contribution can fill the nodal line from the channel of Eq. (27).

(iii) Admixing the tuning direction into $V_{c}=C_{X} X+$ $C_{Y} Y$ adds a first order contribution similar to that in Eq. (30) (Fig. 5)

$$
\begin{aligned}
P_{D \rightarrow A}^{(1, c)}(t) & =\left.\left.\sum_{n_{X}=1}^{\infty}\right|_{A}\left\langle n_{X} 0\left|C_{X} X \sigma_{x}\right| 00\right\rangle_{D}\right|^{2} \\
& \times \frac{\sin ^{2}\left(n_{X} \Omega t / 2\right)}{\left(n_{X} \Omega / 2\right)^{2}} .
\end{aligned}
$$

Here, due to the equidistant position of the $V_{D}$ and $V_{A}$ minima from the origin, the matrix element ${ }_{A}\left\langle 00\left|C_{X} X \sigma_{x}\right| 00\right\rangle_{D}=0$ and $n_{X}$ runs from 1 rather than from 0 as in Eq. (30). Also, due to this symmetry we expect all integrals ${ }_{A}\left\langle n_{X} 0\left|C_{X} X \sigma_{x}\right| 00\right\rangle_{D}$ with even $n_{X}$ to contribute insignificantly. Since $n_{X}>0$, the resonance condition cannot be satisfied in Eq. (33), and the population transfer is similar to that in Eq. (27). For the nodal line, this channel has the same effect as the $\Delta_{12} \neq 0$ channel [Eq. (30)].

\section{B. Interaction with environment}

The full Hamiltonian (11) for the symmetric setup can be written as $H=H_{0}+V$ with

$$
\begin{aligned}
H_{0} & =H_{\mathrm{B}}+H_{\mathrm{S}}-\sigma_{x} C_{Y} Y, \\
V & =\sigma_{x} C_{Y} Y+H_{\mathrm{SB}} .
\end{aligned}
$$

Adding the environment does not affect the interelectronic transition in the first order of TDPT (Fig. 6a)

$$
P_{D \rightarrow A}^{(1)}(t)=\left|{ }_{A}\left\langle 01 \mathbf{n}\left|C_{Y} Y \sigma_{x}\right| 00 \mathbf{n}\right\rangle_{D}\right|^{2} \frac{\sin ^{2}(\Omega t / 2)}{(\Omega / 2)^{2}} .
$$

The effect of $H_{\mathrm{SB}}$ on the donor-acceptor transition appears only in the second order

$$
\begin{aligned}
P_{D \rightarrow A}^{(2, \pm)}(t) & =\mid \int_{0}^{t} d \tau \int_{0}^{\tau} d \tau^{\prime}\left\{{ }_{A}\left\langle 00 \mathbf{n}^{ \pm}\left|C_{Y} Y \sigma_{x}\right| 01 \mathbf{n}^{ \pm}\right\rangle_{D}\right. \\
& \times{ }_{D}\left\langle 01 \mathbf{n}^{ \pm}\left|\lambda_{j Y} Y Q_{j} \mathbf{1}_{2}\right| 00 \mathbf{n}\right\rangle_{D} e^{-i \Omega \tau-i\left(\Omega \pm \Omega_{j}\right) \tau^{\prime}} \\
& +{ }_{A}\left\langle 00 \mathbf{n}^{ \pm}\left|\lambda_{j Y} Y Q_{j} \mathbf{1}_{2}\right| 01 \mathbf{n}\right\rangle_{A} e^{-i \Omega \tau^{\prime}-i\left(\Omega \pm \Omega_{j}\right) \tau} \\
& \left.\times{ }_{A}\left\langle 01 \mathbf{n}\left|C_{Y} Y \sigma_{x}\right| 00 \mathbf{n}\right\rangle_{D}\right\}\left.\right|^{2}
\end{aligned}
$$

where $\mathbf{n}^{ \pm}$and $\mathbf{n}$ differ only by the number of vibrational quanta along the $Q_{j}$ mode: $n_{j}^{ \pm}=n_{j} \pm 1$. Figure $6 \mathrm{~b}$ illustrates the two components of the integrand sum in Eq. (37) as two pathways involving energy transfer between the subsystem and environemnt. These pathways do not require altering the parity of the nuclear wavefunction along the $Y$ coordinate, and thus, their contributions do not form the $Y=0$ nodal line. Therefore, if the transfer due to Eq. (37) is significant compare to that due to Eq. (36), the $Y=0$ nodal line in the subsystem wave-packet will disappear and the donor-acceptor population transfer will be enhanced. Presence of an extra oscillating exponential factor in Eq. (37) indicates that to have an efficient population transfer along this channel the bath frequency $\Omega_{j}$ should be close to the coupling coordinate frequency $\Omega$.

The difference between pathways with and without bath involvement is very similar to that between onephoton absorption and Raman scattering: here, an electronic donor-acceptor transition can be seen as an absorption of a photon, and energy exchange with the bath is analogous to photon scattering. Also, due to the scattering mechanism the normal dipole selection rules that require changing the parity of the nuclear wave-function are altered in the Raman process.

The subsystem-bath interaction through $X$ coordinate $\left(\lambda_{j X} X Q_{j} \mathbf{1}_{2}\right)$ does not appear in the lowest orders of TDPT for the donor-acceptor transfer probabilities, and therefore, these terms do not appreciably change the nuclear dynamics in the diabatic representation. 

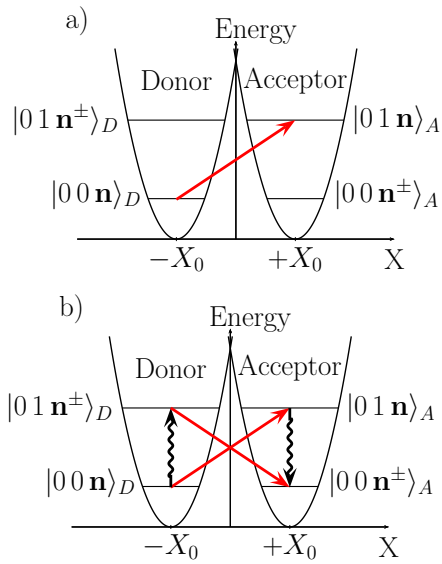

FIG. 6. Main channels for population transfer in the diabatic representation for the subsystem interacting with environment: a) the first order of TDPT Eq. (36), b) the second order of TDPT Eq. (37).

\section{RESULTS AND DISCUSSION}

\section{A. Isolated subsystem}

GP effects are most prominent in the setup where destructively interfering parts of the nuclear wave-packet have equal amplitudes ${ }^{4}$. This is the case for the symmetric setup defined in Sec. III A. In what follows we address the question whether GP effects survive if we break the symmetry between the transfer paths by altering the parameters of the subsystem Hamiltonian (12). Staying within the isotropic $\Omega_{X}=\Omega_{Y}=\Omega$ case there are two scenarios of symmetry breaking with non-symmetric tunnelling paths: i) making $\mathbf{C}$ and $\mathbf{G}$ non-orthogonal : $\mathbf{C} \cdot \mathbf{G} \neq 0$ [case (iii) in Sec. IV A], ii) setting $\Delta_{12} \neq 0$ [case (ii) in Sec. IV A]. We do not consider $\Delta$ variations because they do not cause symmetry breaking between the transfer paths, also their effect has been studied previously. ${ }^{4}$ All other Hamiltonian parameters have been assigned the following values: $\Omega=2, \mathbf{R}_{0}=(3 / 2,0)$, $\Delta=0$.

(i) $\mathbf{C} \cdot \mathbf{G} \neq 0$ : Starting from the symmetric setup we change the angle between the vectors $\mathbf{C}$ and $\mathbf{G}$ by increasing the component $C_{X}$ of the vector $\mathbf{C}$ while keeping $C_{Y}$ constant. According to our qualitative analysis, Sec. IV A, $C_{X}$ does not appreciably affect the dynamics in the diabatic representation. However, in the adiabatic representation, $C_{X}$ component deepens the potential wells resulting in the suppression of the population transfer in simulations without GP (Fig. 7). Thus, although the non-orthogonality between $\mathbf{C}$ and $\mathbf{G}$ preserves the difference between population dynamics with and without GP, it reduces that difference for initial times.

In the symmetric setup with GP there is clear nodal structure of the part of a density tunneled through the CI (Fig. 3a). To clearly observe the nodal pattern in simula-

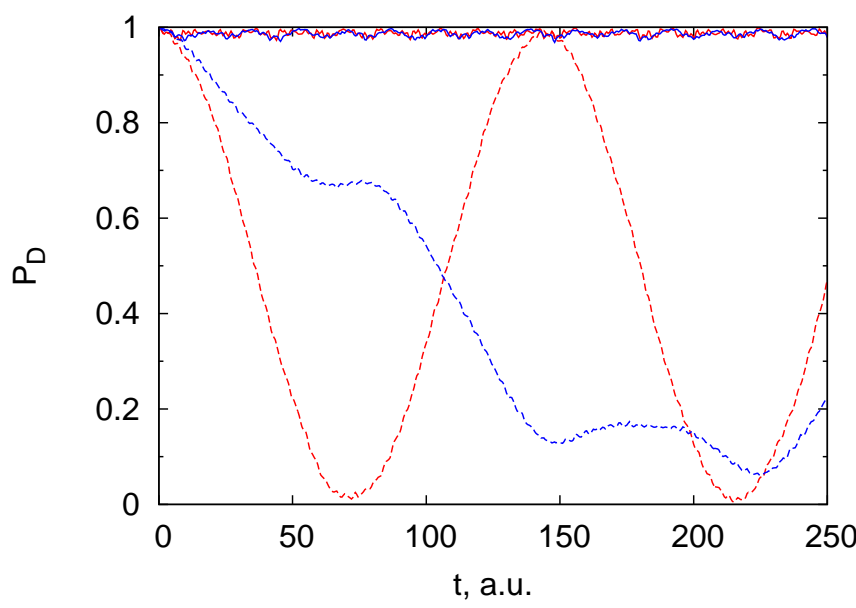

FIG. 7. The subsystem donor well population dynamics $P_{D}(t)$ for different values of $C_{X}$ and $C_{Y}=4$ : (solid red) $C_{X}=0$ with GP, (solid blue) $C_{X}=2$ with GP, (dashed red) $C_{X}=0$ without GP, (dashed blue) $C_{X}=2$ without GP. a)

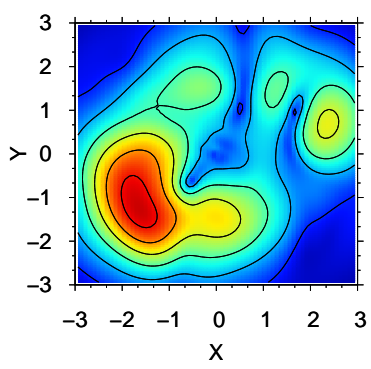

b)

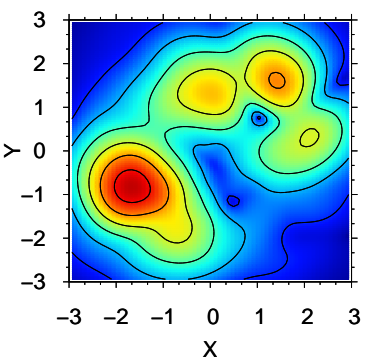

FIG. 8. Snapshots of $\left\langle\phi_{1}^{\text {adi }}\left|\rho_{S}(t)\right| \phi_{1}^{\text {adi }}\right\rangle^{1 / 4}$ at $t=15.0$ a.u. for a non-symmetric configuration $C_{X}=2, C_{Y}=6$ : a) with GP, b) without GP.

tions with non-zero $C_{X}$, we also increased $C_{Y}$ to facilitate the transfer. As follows from Fig. 8 and our qualitative analysis increasing $C_{X}$ destroys the node almost completely. A distinct nodal line "dissolves" in a seemingly chaotic interference pattern of a time-dependent density. At the same time, there are still differences in population dynamics between models with and without GP as $C_{X}$ increases. Thus, the nodal line, which is frequently considered as manifestation of GP, is not always a reliable indicator of GP significance.

(ii) $\Delta_{12} \neq 0$ : The non-zero off-diagonal coupling constant $\Delta_{12}$ shifts the zero coupling line. Following the zero coupling line, the CI point moves out of the line connecting two potential minima for $\Delta_{12} \neq 0$. This opens another transfer channel [Eq. (30)] that facilitates the transfer. For this new channel GP effects are irrelevant and thus, when this channel becomes dominant the population dynamics with and without GP become similar. To illustrate this idea we gradually increase the value of $\Delta_{12}$ from 0 to 0.8 a.u. Simulations show (Fig. 9) that 




FIG. 9. The subsystem donor well population dynamics $P_{D}(t)$ between equivalent wells for several values of $\Delta_{12}$. a)

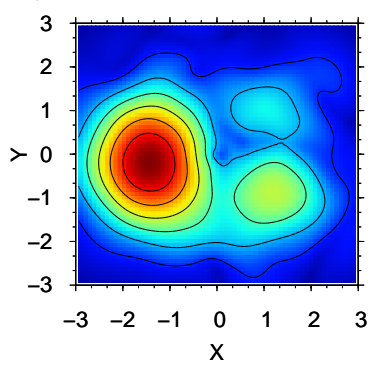

c)

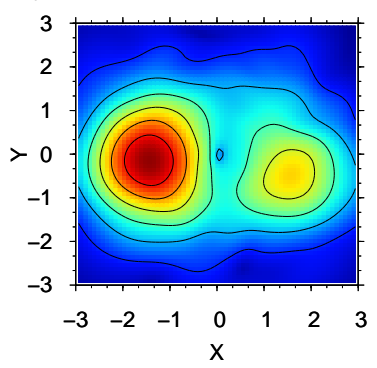

b)

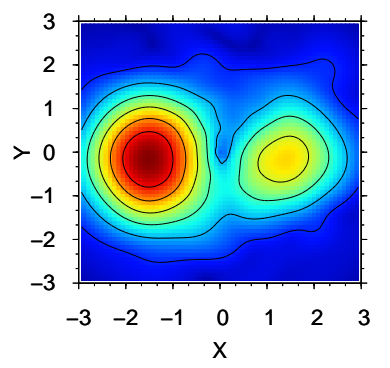

d)

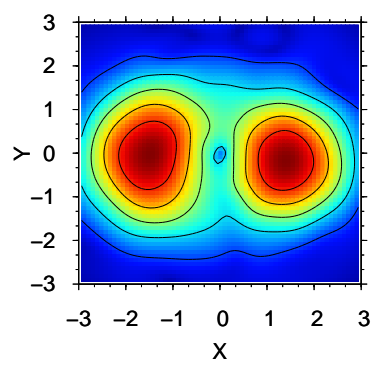

FIG. 10. Snapshots of $\left\langle\phi_{1}^{\text {adi }}\left|\rho_{S}(t)\right| \phi_{1}^{\text {adi }}\right\rangle^{1 / 4}$ for a nonsymmetric configuration $C=6, \Delta_{12}=0.6$ : a) $t=15$ a.u. with GP, b) $t=15$ a.u. without GP, c) $t=30$ a.u. with GP, and d) $t=30$ a.u. without GP.

GP effects are reduced with increasing of $\Delta_{12}$.

The nodal line in this set up may form temporarily for the initial GP dynamics, but does not appear later (Fig. 10). The reason is that the non-nodal channel [Eq. (30)] provides the populations transfer to fill the node. In this case the disappearance of the node correlates well with the reduction of GP significance in the population dynamics.

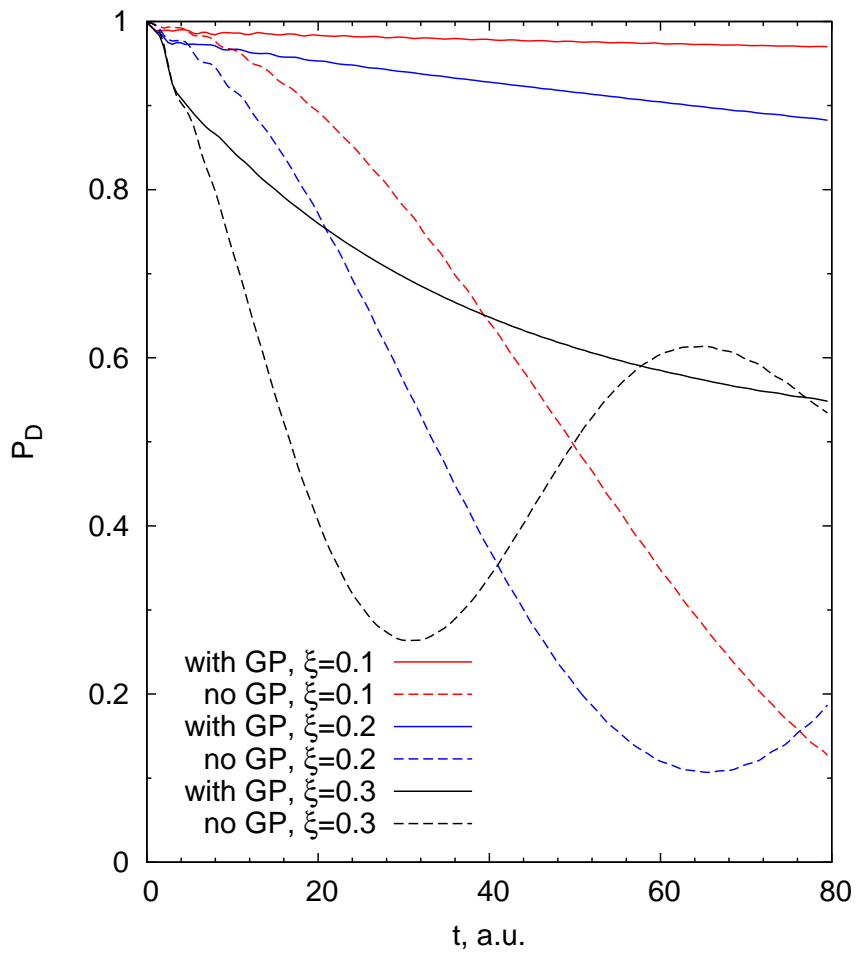

FIG. 11. The subsystem donor well population dynamics $P_{D}(t)$ for $\lambda_{j Y} \neq 0$ and $\Omega_{c}=3.5$.

\section{B. Interaction with environment}

To observe environmental effects in the most clear settings we consider the symmetric setup for the subsystem with $\Omega=2, C=3$, and $X_{0}=1.5$ in a.u., and two environment-subsystem interaction scenarios: bath modes are coupled to either the $X$ or the $Y$ coordinate.

(i) Bath is coupled to $Y\left(\lambda_{j X}=0\right)$ : Figure 11 illustrates the importance of the GP effects for the donor well population $P_{D}$ at various subsystem-bath coupling strengths set with the Kondo parameter $\xi$. In both simulation schemes with and without GP, the initial rate of the population transfer increases with the coupling strength. In the diabatic simulations this is a result of the new population transfer pathway [see Eq. (37) and Fig. 6b] that is opened because of the subsystem-bath interaction. In the adiabatic representation, introducing the subsystem-bath couplings can be seen as a modification of the subsystem Hamiltonian that involves random fluctuations changing the transition barrier heights on the lower adiabatic surface. These barrier fluctuations increase the initial transfer rate. On the other hand, increasing the subsystem-bath coupling strength decreases the amplitude of the population transfer in the adiabatic representation. We attribute this effect to faster decoherence that spreads the subsystem wave packet under the influence of the environment.

According to our qualitative analysis [Eq. (37), Fig. 6b], the $Y=0$ nodal line disappears when the bath 
a)

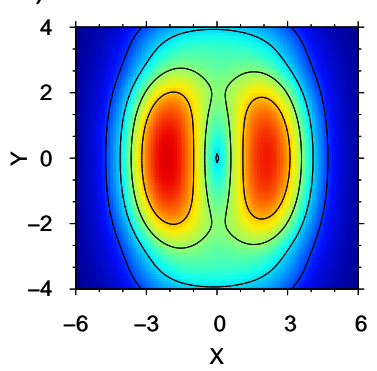

b)

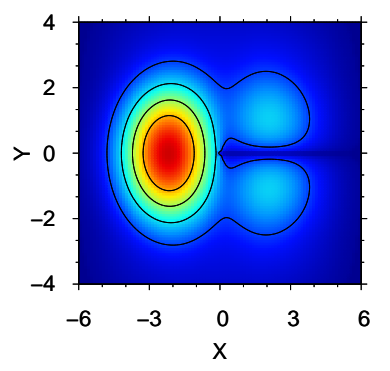

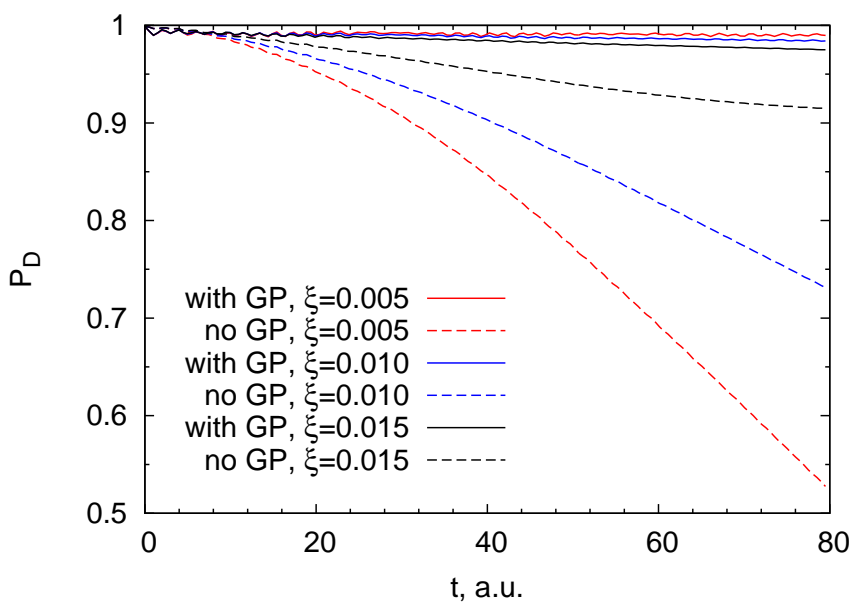

FIG. 13. The subsystem donor well population dynamics $P_{D}(t)$ for small values of the Kondo parameter with $\lambda_{j X} \neq 0$ and $\Omega_{c}=3.5$.

with its environment.

For the subsystem dynamics, GP effects are the most pronounced in the symmetric configuration: when the tuning and coupling directions are mutually orthogonal and two electronic state minima do not have energy difference. For this configuration, the nodal line in the adiabatic density distribution appearing in the course of nuclear dynamics is usually considered as the main GP signature. All subsystem configurations that break symmetry equivalence of the two population transfer pathways do not produce the nodal line. However, we found that even in symmetry broken configurations GP creates substantial difference in the population dynamics. Only if symmetry is broken by increasing the constant interelectronic coupling $\Delta_{12}$ the GP influence can be reduced.

For the symmetric subsystem configuration we have considered two subsystem-bath coupling schemes: all modes of the bath are bi-linearly coupled with either the tuning or the coupling coordinate of the subsystem. The population dynamics with and without GP are quite different for both subsystem-bath coupling schemes. Therefore, we conclude that the GP effects can survive in a large multidimensional molecular system. The main difference between the two coupling schemes is that the nodal line in the subsystem density disappears after including the coupling to the bath along the coupling direction and is preserved in the coupling scheme involving the tuning direction.

To summarize, we would like to emphasize that the loss of the nodal line in subsystem nuclear dynamics does not necessarily mean insignificance of GP effects, and that the most straightforward and accurate way to assess the GP impact is to compare the time evolution of the quantity of interest in dynamics with and without GP. mensional case has been analyzed in two steps: 1) for the isolated subsystem, and 2) for the subsystem interacting 


\section{ACKNOWLEDGMENTS}

We are grateful to R. Kapral and C.-Y. Hsieh for stimulating discussions. This work was supported by Natural Sciences and Engineering Research Council of Canada through the Discovery Grants Program, and the European Union Seventh Framework Programme (FP7/20072013) under grant agreement PIOF-GA-2012-332233.

\section{Appendix A: The effective modes construction}

Here we detail steps of the Hamiltonian transformation starting from Eq. (1) and leading to Eq. (11).

a. Coordinate translation. - We apply a coordinate translation: $q_{j}=x_{j}-\frac{\kappa_{j}+\tilde{\kappa}_{j}}{2 \omega_{i}^{2}}$ to the LVC Hamiltonian, Eq. (1), and obtain

$$
\begin{gathered}
H_{1}=\sum_{j}^{N} \frac{1}{2}\left(p_{j}^{2}+\omega_{j}^{2} x_{j}^{2}\right) \mathbf{1}_{2}+\left(\begin{array}{cc}
-d_{j} x_{j} & c_{j} x_{j} \\
c_{j} x_{j} & d_{j} x_{j}
\end{array}\right) \\
+\left(\begin{array}{cc}
-\Delta / 2 & \Delta_{12} \\
\Delta_{12} & \Delta / 2
\end{array}\right)
\end{gathered}
$$

where

$$
\begin{aligned}
\Delta & =\sum_{j}^{N} \frac{\kappa_{j}^{2}-\tilde{\kappa}_{j}^{2}}{2 \omega_{j}^{2}}+\delta, \\
\Delta_{12} & =\sum_{j}^{N} c_{j} \frac{\kappa_{j}+\tilde{\kappa}_{j}}{2 \omega_{j}^{2}}, \\
d_{j} & =\frac{\tilde{\kappa}_{j}-\kappa_{j}}{2} .
\end{aligned}
$$

b. Subsystem-bath separation.- We define a new set of coordinates $\left\{\tilde{x}_{1}, \tilde{x}_{2}, \ldots\right\}$ obtained from $\left\{x_{1}, x_{2}, \ldots\right\}$ by an orthogonal transformation $\mathbf{O}_{1}: \tilde{\mathbf{x}}=\mathbf{O}_{1} \mathbf{x}$, we use hereafter bold letters as the vector of their corresponding indexed quantities. $\tilde{x}_{1}$ and $\tilde{x}_{2}$ are the subsystem coordinates given by

$$
\begin{aligned}
& \tilde{x}_{1}=\mathbf{e}_{d} \cdot \mathbf{x}, \\
& \tilde{x}_{2}=\left(\mathbf{c} \cdot \mathbf{x}-\tilde{c}_{1} \mathbf{e}_{d} \cdot \mathbf{x}\right) / \tilde{c}_{2},
\end{aligned}
$$

where

$$
\begin{aligned}
& \mathbf{e}_{d}=\mathbf{d} /\|\mathbf{d}\|, \\
& \tilde{c}_{1}=\mathbf{c} \cdot \mathbf{e}_{d}, \\
& \tilde{c}_{2}=\sqrt{\|\mathbf{c}\|^{2}-\left(\mathbf{c} \cdot \mathbf{e}_{d}\right)^{2}} .
\end{aligned}
$$

Thus, the two first rows of $\mathbf{O}_{1}$ are

$$
\left(\begin{array}{c}
\mathbf{e}_{d}^{T} \\
\left(\mathbf{c}^{T}-\tilde{c}_{1} \mathbf{e}_{d}^{T}\right) / \tilde{c}_{2}
\end{array}\right) .
$$

We also define $\tilde{d}_{1}=\|\mathbf{d}\|$ for the later use. The other coordinates, $\left\{\tilde{x}_{j}, j=3, \ldots, N\right\}$, are the bath coordinates, and are defined by the other rows of $\mathbf{O}_{1}$. We employ the Gram-Schmidt orthogonalization procedure to obtain them. New coordinates $\left\{\tilde{x}_{1}, \tilde{x}_{2}, \ldots\right\}$ allows us to write the Hamiltonian as

$$
\begin{aligned}
H_{2}= & \frac{1}{2}\left(\sum_{j=1}^{N} \tilde{p}_{j}^{2}+\tilde{\mathbf{x}}^{T} \boldsymbol{\Lambda} \tilde{\mathbf{x}}\right) \mathbf{1}_{2}+\left(\begin{array}{cc}
-\Delta / 2 & \Delta_{12} \\
\Delta_{12} & \Delta / 2
\end{array}\right) \\
& +\left(\begin{array}{cc}
-\tilde{d}_{1} \tilde{x}_{1} & \tilde{c}_{1} \tilde{x}_{1}+\tilde{c}_{2} \tilde{x}_{2} \\
\tilde{c}_{1} \tilde{x}_{1}+\tilde{c}_{2} \tilde{x}_{2} & \tilde{d}_{1} \tilde{x}_{1}
\end{array}\right),
\end{aligned}
$$

where $\boldsymbol{\Lambda}$ is the Hessian matrix of both diabatic states. In general, $\boldsymbol{\Lambda}$ is non-diagonal in both subsystem and bath subspaces.

c. Diagonalization of subsystem and bath Hessians.We diagonalize the blocks of the Hessian matrix $\boldsymbol{\Lambda}$ corresponding to the subsystem and the bath coordinates. This transformation leads to new subsystem $\{X, Y\}$ and bath $\left\{Q_{j}, j=1,2, \ldots, N-2\right\}$ coordinates. The total Hamiltonian can be split into three parts

$$
H_{3}=H_{\mathrm{S}}+H_{\mathrm{SB}}+H_{\mathrm{B}},
$$

where

$$
\begin{aligned}
H_{\mathrm{S}} & =\frac{1}{2}\left(P_{X}^{2}+\Omega_{X}^{2} X^{2}+P_{Y}^{2}+\Omega_{Y}^{2} Y^{2}\right) \mathbf{1}_{2} \\
+ & \left(\begin{array}{cc}
-\Delta / 2 & \Delta_{12} \\
\Delta_{12} & \Delta / 2
\end{array}\right)+\left(\begin{array}{cc}
-G_{X} X-G_{Y} Y & C_{X} X+C_{Y} Y \\
C_{X} X+C_{Y} Y & G_{X} X+G_{Y} Y
\end{array}\right), \\
H_{\mathrm{B}} & =\frac{1}{2} \sum_{j=1}^{N-2}\left(P_{j}^{2}+\Omega_{j}^{2} Q_{j}^{2}\right) \mathbf{1}_{2}, \\
H_{\mathrm{SB}} & =\sum_{j=1}^{N-2}\left(\lambda_{j X} X+\lambda_{j Y} Y\right) Q_{j} \mathbf{1}_{2} .
\end{aligned}
$$

Here, all non-adiabatic couplings are confined in the subsystem Hamiltonian $H_{\mathrm{S}}$ and coefficients $G_{X / Y}$ and $C_{X / Y}$ are obtained from $\tilde{d}_{1}, \tilde{c}_{1}, \tilde{c}_{2}$ [Eq. (A5)] by the orthogonal transformation of the subspace coordinate. The environment part $H_{\mathrm{B}}$ constitutes a harmonic bath that interacts with the subsystem $H_{\mathrm{S}}$ by $H_{\mathrm{SB}}$. $H_{\mathrm{SB}}$ has simple bilinear terms with coupling constants $\lambda_{j X}$ and $\lambda_{j Y}$ that are independent of electronic state. $H_{\mathrm{S}}$ can be further simplified by completing the squares in $X$ and $Y$ coordinates, introducing the minima $X_{0}=G_{X} / \Omega_{X}^{2}$ and $Y_{0}=G_{Y} / \Omega_{Y}^{2}$, and neglecting a global energy shift

$$
H_{\mathrm{S}}=T_{\mathrm{S}} \mathbf{1}_{2}+\left(\begin{array}{cc}
V_{A} & V_{c} \\
V_{c} & V_{D}
\end{array}\right),
$$

where $T_{\mathrm{S}}, V_{A}, V_{D}$, and $V_{c}$ are defined by Eqs. (15-18) given in the main text.

${ }^{1} \mathrm{~A}$. Migani and M. Olivucci, in Conical Intersection Electronic Structure, Dynamics and Spectroscopy, edited by W. Domcke, D. R. Yarkony, and H. Köppel (World Scientific, New Jersey, 2004) p. 271.

${ }^{2}$ M. V. Berry, P. R. Soc. A 392, 45 (1984).

${ }^{3}$ C. A. Mead and D. G. Truhlar, J. Chem. Phys. 70, 2284 (1979).

${ }^{4}$ I. G. Ryabinkin and A. F. Izmaylov, "Geometric phase effects in dynamics near conical intersections: Symmetry breaking and spatial localization," (submitted) arXiv:1306.6387v2.

${ }^{5}$ L. Blancafort, P. Hunt, and M. A. Robb, J. Am. Chem. Soc. 127, 3391 (2005). 
${ }^{6}$ L. Blancafort, F. Jolibois, M. Olivucci, and M. A. Robb, J. Am. Chem. Soc. 123, 722 (2001).

${ }^{7}$ A. F. Izmaylov, D. Mendive-Tapia, M. J. Bearpark, M. A. Robb, J. C. Tully, and M. J. Frisch, J. Chem. Phys. 135, 234106 (2011).

${ }^{8}$ A. Ferretti, G. Granucci, A. Lami, M. Persico, and G. Villani, J. Chem. Phys. 104, 5517 (1996).

${ }^{9}$ J. Schön and H. Köppel, Chemical Physics Letters 231, 55 (1994).

${ }^{10}$ J. Schön and H. Köppel, J. Chem. Phys. 103, 9292 (1995).

${ }^{11}$ S. C. Althorpe, T. Stecher, and F. Bouakline, J. Chem. Phys. 129, 214117 (2008).

${ }^{12}$ B. Kendrick, J. Chem. Phys. 104, 7475 (1996).

${ }^{13}$ F. Bouakline, S. C. Althorpe, P. Larregaray, and L. Bonnet, Mol. Phys. 108, 969 (2010).

${ }^{14}$ J. C. Juanes-Marcos, S. C. Althorpe, and E. Wrede, Science 309, 1227 (2005).

${ }^{15}$ A. Kelly and R. Kapral, J. Chem. Phys. 133, 084502 (2010).

${ }^{16}$ A. Carollo, I. Fuentes-Guridi, M. F. Santos, and V. Vedral, Phys. Rev. Lett. 90, 160402 (2003).

${ }^{17}$ F. C. Lombardo and P. I. Villar, Phys. Rev. A 87, 032338 (2013).

${ }^{18}$ H. Köppel, W. Domcke, and L. S. Cederbaum, "Multimode Molecular Dynamics Beyond the Born-Oppenheimer Approximation," (John Wiley \& Sons, Inc., 1984) Chap. 2, pp. 59-246.

${ }^{19}$ Q. Meng and H.-D. Meyer, J. Chem. Phys. 138, 014313 (2013).
${ }^{20}$ Z. Li, M. E.-A. Madjet, and O. Vendrell, J. Chem. Phys. 138, 094313 (2013).

${ }^{21}$ C. Lévêque, A. Komainda, R. Taïeb, and H. Köppel, J. Chem. Phys. 138, 044320 (2013).

${ }^{22}$ H. Köppel, J. Gronki, and S. Mahapatra, J. Chem. Phys. 115, 2377 (2001).

${ }^{23}$ C. S. M. Allan, B. Lasorne, G. A. Worth, and M. A. Robb, J. Phys. Chem. A 114, 8713 (2010).

${ }^{24}$ L. Cederbaum, E. Gindensperger, and I. Burghardt, Phys. Rev. Lett. 94, 113003 (2005).

${ }^{25}$ I. Burghardt, E. Gindensperger, and L. S. Cederbaum, Mol. Phys. 104, 1081 (2006).

${ }^{26}$ E. Gindensperger, I. Burghardt, and L. S. Cederbaum, J. Chem. Phys. 124, 144103 (2006).

${ }^{27}$ H.-P. Breuer and F. Petruccione, eds., The Theory of Open Quantum Systems (Oxford University Press, New York, 2002).

${ }^{28}$ See EPAPS Document No. XXX for basis set details. This document can be reached via a direct link in the online article HTML reference section or via the EPAPS homepage (http://www.aip.org/pubservs/epaps.html).

${ }^{29}$ N. Makri, J. Phys. Chem. B 103, 2823 (1999).

${ }^{30}$ A. Altland and B. D. Simons, Condensed Matter Field Theory (Cambridge University Press, New York, 2006). 